\title{
Evaluation of some Methods for Preparing Gliclazide- $\beta$-Cyclodextrin Inclusion Complexes
}

\author{
NP Sapkal, ${ }^{1^{*}}$ VA Kilor, ${ }^{1}$ KP Bhusari ${ }^{2}$ and AS Daud ${ }^{3}$ \\ ${ }^{1}$ Gurunanak College of Pharmacy, Near Dixit Nagar, Nari, Nagpur-440024. INDIA. ${ }^{2}$ Sharad Pawar College of \\ Pharmacy, Wanadongri, Nagpur. ${ }^{3}$ Zim Laboratories Ltd. Kalmeshwar, Nagpur.
}

\begin{abstract}
Purpose: Gliclazide has been found to form inclusion complexes with $\beta$ - cyclodextrin $(\beta-C D)$ in solution and in solid state. The present study was undertaken to determine a suitable method for scaling up gliclazide- $\beta-C D$ inclusion complex formation and to evaluate the effect of some parameters on the efficiency of complexation.

Method: The solid inclusion complexes of gliclazide and $\beta$-cyclodextrin were prepared at a molar ratio of 1:1 and 1:2 by mixing, kneading, and coprecipitation methods both on small and large scales. The effect of parameters such as kneading time and temperature on complexation was also studied. Characterization was performed using infrared spectroscopy, X-ray diffractometry, and dissolution studies. In vitro release studies were carried out in phosphate buffer (pH 6.8).

Result: All the methods of preparation of complexes were found to be useful in increasing the solubility of gliclazide except mixing method where the rise in solubility was not significant. Both kneading and co-precipitation methods in 1:2 molar ratios were found to be equally effective in improving the solubility of gliclazide. The formation of inclusion complexes was evident in these formulations as shown by IR and XRD studies. But when carried out on a large scale, co-precipitation method was found to be more tedious and time-consuming than kneading method. Moreover percent recovery of complexes in the kneading method was found to be $98.76 \%$ as compared to $92.05 \%$ in case of co-precipitation method.

Conclusion: Drug content studies, IR spectroscopic studies, X-Ray diffractometry studies and in vitro dissolution study data indicated that inclusion complexes prepared by kneading method in 1:2 molar ratios were suitable for improving the solubility of gliclazide. The same formulation was prepared at large scale and optimum formulation conditions were established.
\end{abstract}

Keywords: Gliclazide, Inclusion complexes, $\beta$-cyclodextrin, Kneading

*Corresponding Author: E-mail: nidhi_sapkal@yahoo.co.in. Tel: +91-9422815111 


\section{INTRODUCTION}

Cyclodextrins (CDs) are cyclic oligosaccharides, containing a minimum of six D-(+)-glycopyranose units attached by $\alpha-1,4-$ linkages produced by the action of the cyclodextrin-trans-glycosidase enzyme on a medium containing starch. It has a hydrophobic central cavity and a hydrophilic outer surface ${ }^{1}$. CDs have been found to be very useful in enhancing the solubility of poorly water-soluble drugs owing to the formation of inclusion complex of the drug in its hydrophobic cavity $^{2-6}$. The most common natural CDs are $\alpha$-cyclodextrin, $\beta$-cyclodextrin and $\gamma$-cyclodextrin, which are formed by six, seven, and eight glucose units, respectively. Apart from these naturally occurring CDs, various derivatives are also available ${ }^{7,8}$ which may produce better solubility when complexed $^{9,10}$ but cost and toxicity factors poses limitation in their use. Amongst the various available CDs, $\beta$-cyclodextrins $(\beta-C D)$ are the cheapest and are nontoxic for oral use $^{11}$.

Gliclazide (Glz) is a second-generation sulphonylurea oral hypoglycemic agent used in the treatment of non-insulin dependent diabetes mellitus. It stimulates insulin secretion by pancreatic beta cells. In the long-term, it reduces hepatic gluconeogenesis, and increases insulin effects by acting at receptor or post-receptor sites. It also inhibits platelet aggregation and increases fibrinolysis ${ }^{12}$. But the problem with this potentially useful hypoglycemic agent is that it is practically insoluble in water. This limits its bioavailability and may be the reason for its delayed absorption ${ }^{13}$.

Since CDs are relatively more water-soluble molecules and $\mathrm{Glz}$ can form inclusion complex with this, it can be used as a solubility enhancer for $\mathrm{Glz}^{14-16}$. Several methods have been proposed to obtain solid drug-CD complexes, both in liquid and in solid medium, but there is still no general rule or universal method, probably because each drug to be entrapped is a special case and optimal conditions depend on the characteristic of both the host and guest molecules. Selection of the most suitable preparation method for a given drug requires careful evaluation because apart from consideration of the performance of the obtained product, such as the dissolution profile, other factors such as simplicity, low cost, high yield, swiftness and ease of scale-up also play a major role in the choice of a method.

The objective of the present study was to compare the physicochemical properties and dissolution profiles of the inclusion complexes of $\mathrm{Glz}-\beta-C D$ prepared by different methods in order to devise a suitable method that is easier, less costly, faster and produces highyield complexes, when scaled up.

\section{EXPERIMENTAL Materials}

Both $\beta$ - Cyclodextrin and Gliclazide were received from Zim Laboratories Ltd., Nagpur, India, as gift samples. Potassium dihydrogen phosphate $(35.084 \mathrm{~g})$ and disodium hydrogen phosphate $(28.80 \mathrm{~g})$ were dissolved in $1000 \mathrm{ml}$ of water to produce phosphate buffer of $\mathrm{pH} 6.8$. All the reagents and solvents were analytical grade, and double distilled water was used.

\section{METHODS}

\section{Phase Solubility Studies}

Phase solubility studies were carried out according to the method reported by Higuchi and Connors ${ }^{17}$. Excess amounts of gliclazide were added to phosphate buffer solutions $(\mathrm{pH}$ 6.8) containing $\beta$-cyclodextrin in different molar concentrations and stirred for four days at constant temperature. The filtered solutions were analyzed on a double beam UV spectrophotometer (SHIMADZU UV2401PC, Kyoto, Japan) at $226 \mathrm{~nm}$, after diluting suitably with phosphate buffer, to define solubility characteristics.

\section{Physical mixing method}

Gliclazide and $\beta-C D$ were blended in the mortar for 30 min in molar ratios of 1:1 and 1:2 (GPM1 and GPM2) the total weight of the mixture was 100g. The physical mixtures were also prepared at large scale by blending gliclazide 
with $\beta-C D$ (in the same ratio) total weight of approx $20 \mathrm{~kg}$ in a rapid mass granulator (GMI, Mumbai, INDIA) for 30mins. These powdered physical mixtures were then stored in the room at controlled temperature $\left(25^{\circ} \pm 2.0^{\circ}\right)$ and humidity conditions (Relative humidity $40-50 \%$ ) for comparison with the corresponding solid complex powders.

\section{Kneading method}

Gliclazide and $\beta-C D$ in the molar ratios of $1: 1$ and 1:2 (GK1 and GK2) were wetted with appropriate quantity of water so as to obtain a paste. Both 1:1 and 1:2 molar ratios were then subjected to different trituration timings ranging from $15 \mathrm{~min}$. to $1 \mathrm{hr}$. This was done to study the effect of trituration time over the effective complexation. After that it was dried at $50^{\circ} \mathrm{C}$, for one day, crushed, sieved and stored at temperature of $25^{\circ} \pm 2.0^{\circ}$ and relative humidity between $40-50 \%$. For small scale mortar and pestle was used while for large scale rapid mass granulator was used. The kneading method was repeated at $75^{\circ} \mathrm{C}$.

\section{Co-precipitation method}

Gliclazide (3.23 g) was dissolved in minimum quantity of acetone and was added drop wise to the solution of $\beta-C D(11.35 \mathrm{~g}$ for GCP1 and $22.7 \mathrm{~g}$ for GCP2) in minimum quantity of water previously maintained at $75^{\circ} \mathrm{C}$ while stirring. Stirring was maintained for $1 \mathrm{~h}$ at $75^{\circ} \mathrm{C}$. Then gradually it was cooled to room temperature while stirring. The precipitates were then filtered, dried and stored at temperature of $25^{\circ}$ $\pm 2.0^{\circ}$ and relative humidity between $40-50 \%$. The experiment was carried out both at small and large scale in 1:1 and 1:2 molar ratios (GCP1 and GCP2).

\section{Drug Content Measurements}

After 3 days of storage, all the powders obtained were assayed spectrophotometrically (Shimadzu, UV2401PC spectrophotometer) at $226 \mathrm{~nm}$ by dissolving in methanol to determine the percent yield of all the methods.

\section{IR Spectroscopy}

Infra red spectrums of the prepared complexes were carried out using FT-IR (Shimadzu FTIR-
8101) based on the $\mathrm{KBr}$ disc method and compared with that of pure drug.

\section{$X$-ray Diffractometry}

The X-ray diffractograms were obtained using on $X-R a y$ diffraction instrument (Philips AnalyticalX'Pert $\mathrm{PRO}$ ) with $\mathrm{Ni}$-filtered $\mathrm{Cu}$ radiation, at a voltage of $40 \mathrm{kV}$ and current of $30 \mathrm{~mA}$. The scanning speed was 2 degree/min between 2 and 40 theta.

\section{Dissolution Rate Studies}

Dissolution studies on the pure drug, and the inclusion complexes prepared by different methods were performed in phosphate buffer (pH6.8) using USP six station dissolution rate test apparatus (VEEGO Scientific, Mumbai, India) with a paddle stirrer. The stirring speed employed was $100 \mathrm{rpm}$, and the temperature was maintained at $37^{\circ} \mathrm{C} \pm 0.5^{\circ} \mathrm{C}$. Powdered samples of each preparation, equivalent to $40 \mathrm{mg}$ of gliclazide, were placed in the dissolution medium. Samples withdrawn at different time intervals were determined for gliclazide content measuring spectrophtometrically wavelength of at $226 \mathrm{~nm}$ after diluting suitably.

\section{RESULTS}

\section{UV Measurements}

The percent yield of GPM1 and GPM2 were found to be in the range of $99.58 \%$ to $98.71 \%$. The complexes prepared by kneading method (GK1 and GK2) were obtained in $98.76 \%$ to $97.52 \%$ yield. While the percent yield of GCP1 and GCP2 was in the range of $92.05 \%$ to $93.24 \%$.

\section{Phase Solubility Studies}

The phase solubility diagram of $G \mid z-\beta-C D$ was obtained by plotting the changes in guest solubility as a function of CD concentration and is shown in Fig. 1. The solubility curve was classified as Bs type according to Higuchi and Connors ${ }^{17}$ indicating the limited solubility of the complex, since the initial rising portion was followed by plateau, and finally the concentration of GLZ decreased with the precipitation of solid microcrystalline complex. 
The extent of complexation in aqueous media is characterized by the apparent stability constant, Ks. In the present study, Ks determination was based on the solubility diagram which required calculations involving drug solubility. Hence, Ks

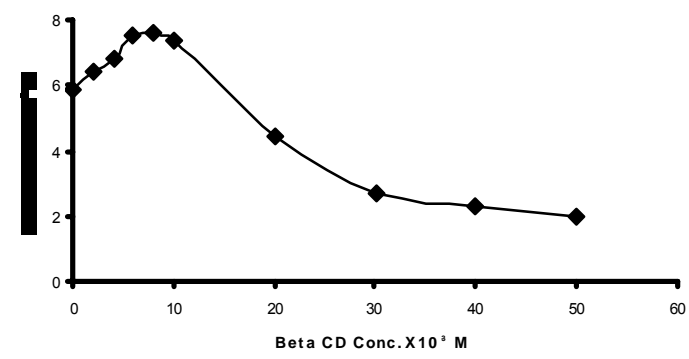

Fig.1: Phase solubility plot for gliclazide in $\beta$-cyclodextrin

$\mathbf{E}$

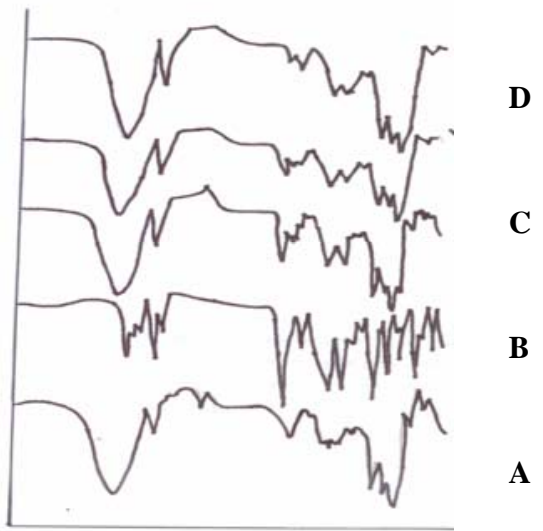

Fig. 2: IR spectra of: $\beta-C D(A), G L Z$ (B), GPM2 (C), GK2 (D) and GCP2 (E).

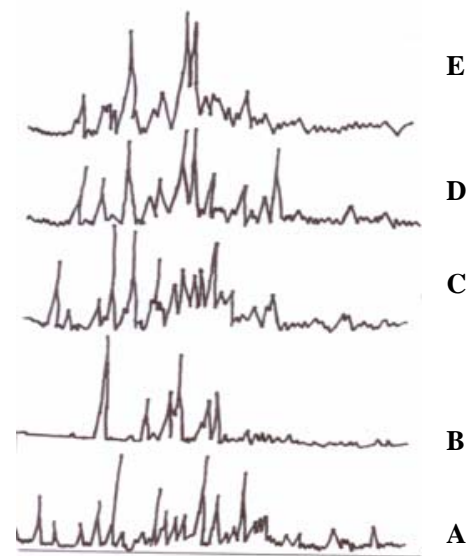

Fig. 3: X-ray diffraction patterns of $\beta-C D(A)$, Pure Glz (B), GPM2 (C), GK2 (D), GCP2 (E). 


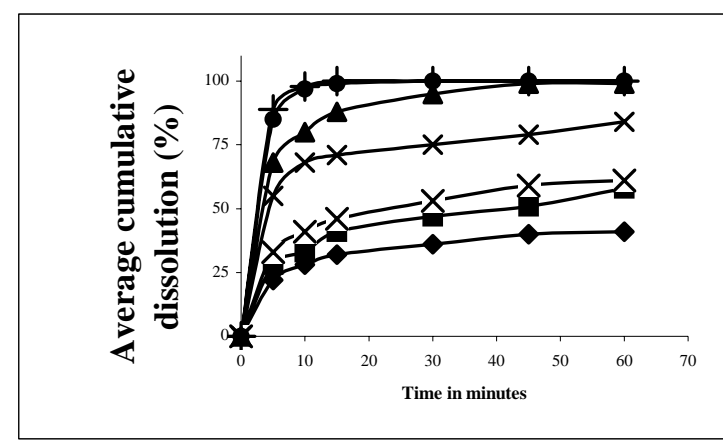

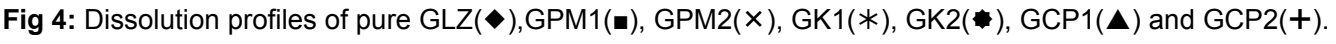

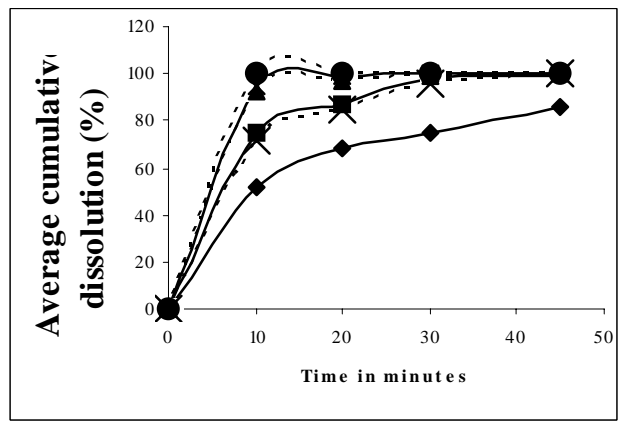

Fig 5: Dissolution profiles of Kneaded complexes: $15 \mathrm{~min}$ kneading at $25^{\circ} \pm 2.0^{\circ}(\diamond)$, 30min kneading at $25^{\circ} \pm 2.0^{\circ}(-), 45 \mathrm{~min}$ kneading at $25^{\circ} \pm 2.0^{\circ}(*), 15 \mathrm{~min}$ kneading at $75^{\circ} \mathrm{C}(--\times--), 30 \mathrm{~min}$ kneading at $75^{\circ} \mathrm{C}\left(--\mathbf{\Delta - - )}, 45 \mathrm{~min}\right.$ kneading at $75^{\circ} \mathrm{C}(---)$

value was calculated according to the equation of Higuchi and Connors ${ }^{17}$ from the initial straightline portion of the solubility diagram.

$$
\mathrm{Ks}=\text { slope } / \mathrm{S}_{\mathrm{o}}(1 \text {-slope })
$$

where $S_{0}$ is the intrinsic solubility of gliclazide in the absence of $\beta-C D$. The value of $K s$ was found to be $81 \mathrm{M}^{-1}$

\section{IR Spectroscopy}

The IR spectra of the complexes together with the pure drug are shown in Fig. 2 for comparison. As can be clearly seen from the spectra, the characteristic bands of gliclazide at 1352, 1438, 1709 and 1597 were modified significantly in the solid complexes as a result of complex formation. The $\mathrm{C}-\mathrm{H}$ stretching peak due to aromatic ring of gliclazide was absent in all of the complexes. The spectra of GPM1 and GPM2 resemble more the spectrum of pure gliclazide. In the case of GK1, GK2, GCP1 and GCP2, the characteristic bands of gliclazide at 1352, 1438, 1709 and 1597 were modified significantly as a result of complex formation. The spectra of all the complexes made on a small scale were similar to the spectra of the corresponding samples made on a large scale.

\section{$X$-Ray Diffractometry}

The X-ray diffraction patterns of pure gliclazide, $\beta-C D$, physical mixtures, as well as kneaded and co-precipitated powders of the GLZ and $\beta-C D$ are represented in Fig. 3. The peak position (angle of diffraction) is an indication of crystal 
structure which peak height is the measure of sample crystallinity.

The diffractograms of pure gliclazide and pure $\beta$ $C D$ exhibited a series of intense peaks, which are indicative of their crystallinity. The diffractogram of the physical mixture was constituted practically by simple superposition of each component, indicating the presence of gliclazide in crystalline state and no formation of a new structure. This result supports the finding of IR spectra. The inclusion complexes prepared by kneading and co-precipitation methods both on small and large scales showed varied reduction in crystallinity based on preparation method used. Intensity of peak sharpness was greater in physical mixtures than in kneaded products and co-precipitates. In the diffractograms of GK1, GK2, GCP1 and GCP2, many of the peaks of gliclazide and $\beta-C D$ disappeared while some are less intense. Moreover, new peaks are also seen in the diffractograms of GK1, GK2, GCP1 and GCP2 indicating formation of new compound.

\section{Dissolution Studies}

The reported values were obtained by calculating the arithmetic mean of five measurements, and standard deviation bars are omitted to avoid overlapping. Fig. 4 shows the dissolution profiles of pure gliclazide, GPM1, GPM2, GK1, GK2, GCP1 and GCP2. While the pure drug is practically insoluble, all other powders show improvement in drug solubility. The extent of this enhancement in the solubility varies with the method of complexation and host-guest molar ratio.

GPM1 and GPM2 showed the lowest improvement in solubility. But the solubility of GPM2 is more than GP1. Co-precipitation method gave better dissolution efficiency than kneading when 1:1 complexes are compared. But none of these dissolution curves show better or equal performance than the dissolution curves of GK2 and GCP2. Powder prepared by kneaded and co-opercipitation method in 1:2 molar ratios are produced.

\section{DISCUSSION}

The stoichiometry of the complexes in the solid phase evaluated on the basis of data in the plateau region of the solubility diagrams and the component ratio was estimated as $1: 2$. These results were confirmed by chemical analysis of the solid complex.

As shown by IR spectroscopy studies it was confirmed that all the methods are effective for the formation of inclusion complexes. However, the spectra of GPM1 and GPM2 resemble more to the spectrum of pure gliclazide. This means that the extent of complexation was less in the complexes made by physical mixting. While complexes made by kneading and coprecipitation method showed better complexation, as their spectra were significantly different from the spectra of pure drug. Modification of the characteristic bands of gliclazide also indicates the formation of complex. Absence of the $\mathrm{C}-\mathrm{H}$ stretching peak of gliclazide confirms that all the methods are effective for the formation of inclusion complexes. But GPM1 and GPM2 were more like the spectrum of pure gliclazide, it means the extent of complexation was less in complexes made by physical mixture. The fact that complexes made by kneading and coprecipitation methods showed greater complexation, is buttered by the significantly different from the spectrum of pure drug.

The inclusion complexes prepared by kneading and co-precipitation methods showed reduction in the crystallinity based on the particular preparation method used. Reduction in the intensity of peak sharpness for kneaded and co precipitated complexes initiate that complexation was practically complete. The disappearance of the peaks of gliclazide and $\beta-C D$ and appearance of new peaks indicates the formation of new compound.

The lower dissolution efficiencies of GPM1 and GPM2 indicate that physical mixing is a less efficient method for complexation. In the case of the physical mixtures, the small rise in solubility when compared to, pure gliclazide is due to the rapid formation of inclusion complexes in the dissolution medium or to the wetting effect of $\beta$ CD. Incidentally, $\beta-C D$ has surfactant-like properties owing to the hydrophilicity of its exterior surface which can lower the interfacial tension between poorly soluble drugs and the dissolution medium, resulting in higher 
dissolution rate. In the case of 1:1 molar complexes prepared by kneading method a lower amount of $\beta-C D$ resulted in partial complexation and hence lower improvement in solubility while co-precipitated 1:1 powders exhibited considerable improvement in solubility proving the efficiency of this method with regard to inclusion complexation.

In the case of complexes prepared in a 1:2 molar ratio, the similar dissolution curves suggests similarity in the nature of complexes prepared by kneading and co-precipitation. The more efficient method may be co-precipitation because it allows the formation of complex when molecules having appropriate energies collide in correct orientation, and this is helped by heat and stirring. As in the case of co-precipitation method, inclusion complex formation takes place at molecular level. Even in kneading method, particles are actually undergoing localized dissolution due to effect because of water and heat (is supplied by vigorous mixing). Therefore, if co-precipitation method forms the inclusion complexes from two fully soluble solutes, after their molecules collide in correct orientation, the kneading method results in inclusion complexes by virtue of the concurrence of several factors during kneading. The presence of water provides the solvent for dissolving the reactants and precipitating the product; the high rate of mixing provides localized heating; sufficient time is required for all of the reactants to react. Thus, the complexes formed by kneading method also result in true complexes like co-precipitated ones if a solvent of suitable nature in appropriate amount is used along with appropriate period of trituration. Moreover, these inclusion compounds made by both kneading and co-precipitation have a low degree of crystallinity and enhances dissolution owing to increased drug particle wettability and formation of a readily soluble inclusion complex in the dissolution medium.

\section{Comparison of Kneading and Co- precipitation method for scaling up}

Since the 1:2 inclusion complexes prepared by both kneading and co-precipitation methods showed similar performance in dissolution studies, further studies were carried out to determine the easier, faster and cost effective method of complexation. While scaling up it was realized that it is far easier to deal with large quantities of solid than with that of liquids. Due to the limited solubility of $C D$, large volumes of water were used. Tank capacity, time and energy for heating and cooling are important cost factors. Treatment and disposal of the mother liquor obtained after collecting the complex is also a concern, but for kneading method mixers like a rapid mixer granulator can be used. It was found that kneading was faster when compared to co-precipitation. The only time-consuming step in kneading was drying, while in the case of co-precipitation, the solution had to be stirred for longer periods in large vessels with the aid of stirrers. If stirring time is reduced, there is reduction in the yield. Moreover, the time taken by large quantities of liquid for cooling is longer more and forced cooling may have effect on the crystallinity of the co-precipitated product. Thus less hardware would be required for kneading method to achieve scale up than for co-precipitated method. For reasons of simplicity, ease and time, further studies were carried out on kneading method only.

Dissolution profiles of the complexes prepared by kneading for 60 minutes both at room and elevated temperatures are not shown in the figure since their release profiles were similar to those of to the complexes prepared in a kneading time of 45 minutes. Since coprecipitation was performed at $75^{\circ} \mathrm{C}$, the aim here was to study the role of temperature in the formation of inclusion complexes. . During these studies all other factors were constant. The only variable was kneading time. It is clear from the studies that kneading time affects the efficiency of inclusion complexation. After 15min the complexation was incomplete. Kneading for a $30 \mathrm{~min}$ period is optimum for complete complexation while triturating for a shorter period will give powders with lower dissolution profile. Triturating for longer time will be wastage of energy.

\section{CONCLUSION}

Beta cyclodextrin can be used to prepare suitable inclusion complexes of gliclazide. All the complexes prepared showed remarkable 
increases in the water solubility of the drug. With increasing quantity of cyclodextrin in the powder, a rise in the level of complexation and drug solubility was obtained. Thus, kneading and co-precipitation methods can be used for the preparation of complexes but kneading method would be more suitable on a large scale.

\section{REFERENCES}

1. Bekers O, Uijtendaal EV, Beijnen JH, Butt A, Underberg WJM. Cyclodextrins in the pharmaceutical field. Drug Dev Ind Pharm 1991; 17 (11): 1503-1549.

2. Ammar HO, El-Nahhas SA, Emara LH. Improvement of some pharmaceutical properties of drugs by cyclodextrin complexation. Part 7: Trimethoprim. Pharmazie 1997; 52 (5): 376-379.

3. Chowdary KPR, Buchi NN. Nimesulide and $\beta$ cyclodextrin inclusion complexes: physicochemical characterization and dissolution rate studies. Drug Dev Ind Pharm 2000; 26 (11): 1217-1220.

4. Dhanraju MD, Kumaran K, Baskaran T, Moorty MS. Enhancement of bioavailability of griseofulvin by its complexation with $\beta$-cyclodextrin. Drug Dev Ind Pharm 1998; 24 (6): 583-587.

5. Hedges AR, Shieh WJ, Sikorski CT. Encapsulation and controlled release of food ingredients. In: Risch SJ, Reineccius GA (eds). ACS Symposium series No. 590 1995, pp 60.

6. Loftsson T, Brewster ME. Pharmaceutical applications of cyclodextrins. 1. Drug solubilization and stabilization. J Pharm Sci 1996; 85(10): 10171025.

7. Bruck SD. Controlled Drug Delivery. Vol.1, CRC Press, Boca Raton, United States, 1983, pp125-148.
8. Atwood JL, Davis JED, MacNicol DD. Inclusion Compounds. Vol.III, Academic Press, London, 1984, pp 333-375.

9. Linares MS, de Bertorello MM, Longhi MR. Effect of hydroxypropyl- $\beta$-cyclodextrin on the solubility of an antibacterial Isoxazolyl-naphthoquinone. Drug Dev Ind Pharm 2000; 26(9): 1003-1006.

10. Moyano JR, Arias-Blanco MJ, Gines JM, Rabasco AM, Perez-Martinez JI. Dissolution behavior of oxazepam in presence of cyclodextrins: Evaluation of oxazepam-Dimeb binary system. Drug Dev Ind Pharm 1997(A); 23 (4): 379-385.

11. Redente E, Szente L, Szejtli J. Cyclodextrin complexes of salts of acidic drugs: Thermodynamic properties, structural features, and pharmaceutical applications. J Pharm Sci 2001; 90(8): 979-986.

12. Dollery SC. Therapeutic Drugs. Churchill Livingstone, London, 1991

13. Parfitt K. Martindale, The complete drug reference. Pharmaceutical Press, London, 1999.

14. Moyano JR, Arias-Blanco MJ, Gines JM, Rabasco AM, Perez-Martinez JI, Mor M, Giordano F. Nuclear magnetic resonance investigations of the inclusion complexation of gliclazide with beta-cyclodextrin. $J$ Pharm Sci 1997(B); 86(1): 72-75.

15. Ozkan Y, Atay T, Dikmen, N, limer A, Aboul Enein HY. Improvement of water solubility and in vitro dissolution rate of gliclazide by complexation with B-cyclodextrin. Int J Pharm 1997; 148 (2): 211-217.

16. Winters $C S$, York $P$, Timmins $P$. Solid state examination of a gliclazide- $\beta$-cyclodextrin complex. Eur $J$ Pharm Sci 1997; 5 (4): 209-215.

17. Higuchi $T$, Connors $K A$. Phase solubility Techniques. Adv Anal Chem Instrum 1965; 4: 117-212. 